\title{
Factors distinguishing invasive from pre- invasive adenocarcinoma presenting as pure ground glass pulmonary nodules
}

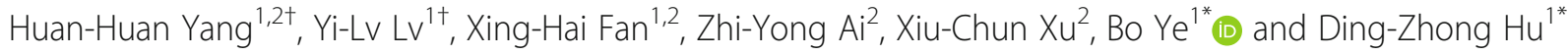

\begin{abstract}
Background: To investigate predictors of pathological invasiveness and prognosis of lung adenocarcinoma in patients with pure ground-glass nodules (pGGNs).

Methods: Clinical and computed tomography (CT) features of invasive adenocarcinomas (IACs) and pre-IACs were retrospectively compared in 641 consecutive patients with pGGNs and confirmed lung adenocarcinomas who had undergone postoperative $\mathrm{CT}$ follow-up. Potential predictors of prognosis were investigated in these patients.

Results: Of 659 pGGNs in 641 patients, 258 (39.1\%) were adenocarcinomas in situ, 265 (40.2\%) were minimally invasive adenocarcinomas, and 136 (20.6\%) were IACs. Respective optimal cutoffs for age, serum carcinoembryonic antigen concentration, maximal diameter, mean diameter, and $\mathrm{CT}$ density for distinguishing pre-IACs from IACs were 53 years, $2.19 \mathrm{ng} / \mathrm{mL}, 10.78 \mathrm{~mm}, 10.09 \mathrm{~mm}$, and -582.28 Hounsfield units (HU). Univariable analysis indicated that sex, age, maximal diameter, mean diameter, CT density, and spiculation were significant predictors of lung IAC. In multivariable analysis age, maximal diameter, and $\mathrm{CT}$ density were significant predictors of lung IAC. During a median follow-up of 41 months no pGGN IACs recurred.
\end{abstract}

Conclusions: pGGNs may be lung IACs, especially in patients aged $>55$ years with lesions that are $>1 \mathrm{~cm}$ in diameter and exhibit CT density $>-600 \mathrm{HU}$. pGGN IACs of $<3 \mathrm{~cm}$ in diameter have good post-resection prognoses.

Keywords: Adenocarcinoma, Ground-glass nodule, Computed tomography

\section{Background}

In computed tomography (CT) images pure groundglass nodules (pGGNs) are visualized as homogeneous hazy lesions of the lung in which the vascular and bronchial components are preserved and there is no solid component. The use of CT to screen for lung cancer has resulted in detection of an increasing number of pGGNs [1], which are consistently found to constitute precursors of lung invasive adenocarcinomas (IACs). pGGNs include atypical adenomatous hyperplasia,

\footnotetext{
* Correspondence: yb1373@shchest.org; hu_dz94@hotmail.com

${ }^{\dagger}$ Huan-Huan Yang and Yi-Lv Lv contributed equally to this work.

'Department of ThoracicSurgery, Shanghai Chest Hospital, Shanghai Jiaotong University, Shanghai 200030, China

Full list of author information is available at the end of the article
}

adenocarcinoma in situ (AIS), and minimally invasive adenocarcinoma (MIA) [2-4]. These lesions often grow slowly and have good prognoses [5-8];however, many pGGNs $>1 \mathrm{~cm}$ in diameter eventually develop into IACs, which have a worse prognosis [9] andrequire different therapeutic strategies $[10,11]$. Therefore, the ability to detect these IACs preoperatively on the basis of clinical manifestations and CT characteristics is beneficial. The purpose of the current study was to identify factors that distinguished IACs from MIAs and AISs in patients with pGGNs who had undergone surgical resection, as well as to determine their prognoses.

(c) The Author(s). 2020 Open Access This article is licensed under a Creative Commons Attribution 4.0 International License, which permits use, sharing, adaptation, distribution and reproduction in any medium or format, as long as you give appropriate credit to the original author(s) and the source, provide a link to the Creative Commons licence, and indicate if changes were made. The images or other third party material in this article are included in the article's Creative Commons licence, unless indicated otherwise in a credit line to the material. If material is not included in the article's Creative Commons licence and your intended use is not permitted by statutory regulation or exceeds the permitted use, you will need to obtain permission directly from the copyright holder. To view a copy of this licence, visit http://creativecommons.org/licenses/by/4.0/ The Creative Commons Public Domain Dedication waiver (http://creativecommons.org/publicdomain/zero/1.0/) applies to the data made available in this article, unless otherwise stated in a credit line to the data. 


\section{Materials and methods Study design}

This study was conducted in accordance with the Declaration of Helsinki. The Institutional Review Board and the Ethics Committee of Shanghai Chest Hospital approved the study, and waived the requirement for patient consent due to its retrospective design.

\section{Patients}

A search of the electronic medical records of Shanghai Chest Hospital yielded 5748 consecutive patients with a pathological diagnosis of lung cancer who had undergone surgical resection at the Department of Thoracic Surgery between January 2014 and December 2015. Inclusion criteria were (1) availability of pre-resection CT; (2) lesions manifesting as pGGNs on CT; (3) lesions $<3$ $\mathrm{cm}$ in diameter as determined via CT; and (4) pathologically proven lung adenocarcinoma. Exclusion criteria were: (1) no pre-resection CT available; (2) lesions manifesting as part-solid or solid nodules on CT; (3) lesions $>3 \mathrm{~cm}$ in diameter on CT; and (4) histological diagnosis other than lung adenocarcinoma, such as AAH or squamous cell carcinoma. The following clinical data were recorded for all included patients: age, sex, smoking history (never or current/former), symptoms (none, or any of cough, shortness of breath, fever, hemoptysis, chest pain, recurrent pulmonary infection), serum carcinoembryonic antigen (CEA) concentration, and CT features. All patients underwent preoperative thoracic unenhanced CT examination at our hospital.

CT imaging Lung CT scans were performed with a Somatom Sensation-64 (Siemens Medical Systems, Forchheim, Germany) with $120 \mathrm{kVp}$ and $100 \mathrm{mAs}$. All CT examinations included the entire thorax at full suspended inspiration with the patient lying supine. When a nodule was identified, target $\mathrm{CT}$ was performed with the following parameters: pitch, $0.64 ; 1-3 \mathrm{~s}$ scan time; matrix size, 1024*1024; FOV, $180 \mathrm{~mm}$. The lung window width was consistently 1600 Hounsfield units (HU), and the window level was $-600 \mathrm{HU}$.

Nodule size was expressed as maximal (longest diameter on axial images) and mean (average of the maximum length and width of the nodule) diameters [12]. $\mathrm{CT}$ density was defined as the average $\mathrm{CT}$ attenuation (HU) within the nodule that did not contain blood vessels or bronchioles. Two readers (radiologist J.G. with 16 years of experience in chest CT and surgeon J.F. with 12 years of experience in thoracic surgery) who were blinded to the histopathological results and clinical data evaluated all CT scans independently. Each reader measured the sizes and $\mathrm{d}$ of the lesions in the lung window setting on the transverse $\mathrm{CT}$ section that displayed the largest nodule dimensions. The average of the measurements obtained by each of the two reviewers was used for analysis. The observers also recorded the presence of particular signs such as pleural retraction, air bronchogram, bubble lucency, and spiculated margins. Pleural retraction was defined as linear attenuation heading toward the pleura or the major or minor fissure from a pGGN. Air bronchogram was defined as air-filled bronchi within a pGGN. Bubble lucency was defined as the presence of small spots of round or ovoid air attenuation within a pGGN. Spiculated margins were defined as the presence of strands extending from a nodule margin into the lung parenchyma without reaching the pleural surface [4]. The results were compared between two readers. If the results were discrepant, the two readers reevaluated the scan to reach a consensus. If no consensus was reached, another radiologist (Z.X.G. with 24 years of experience) was consulted and their decision was deemed final.

\section{Histopathological findings}

To ensure that the resected nodules corresponded to nodules observed inCT scans, the radiologic and surgical procedures were conducted on the same day; CT-guided microcoils were inserted to mark both the nodule and the visceral pleural surface. Intra-operative fluoroscopy was then used to identify the microcoils, and thus the nodule to be resected. Two chest pathologists (F.X.J., with 12 years of experience, and Z.H., with 23 years of experience), who were blinded to all clinical information reviewed the pathological specimens independently and classified the lesions as atypical adenomatous hyperplasia, AIS, MIA, or IAC in accordance with the criteria described in the 2015 World Health Organization Classification of Lung Tumors [13, 14];Disagreements were resolved by consensus. They also classified the histological subtypes of IACs as lepidic predominant, acinar predominant, papillary predominant, micropapillary predominant, solid predominant, or invasive mucinous adenocarcinoma and then classified all tumors as preIAC (including AIS and MIA) or IAC.

\section{Statistical analysis}

For continuous predictors, optimal cutoff values were determined via the maximal Youden index (sensitivity + specificity -1). Receiver operating characteristic curves, and corresponding areas under the curves, sensitivities and specificities were presented for these dichotomized predictors using the optimal cutoffs.

Integer cutoffs can be more convenient in clinical practice; thus, approximate integer of optimal cutoffs were applied for maximal and mean diameters (both 10 $\mathrm{mm})$, CT-determined density $(-600 \mathrm{HU})$, and age (55 years). Although our cutoff value for CEA was $2.19 \mu \mathrm{g} / \mathrm{L}$, we used the upper limit of the normal range of $5 \mu \mathrm{g} / \mathrm{L}$ as our standard for stratification. 
These dichotomized predictors were described using frequencies and percentages, differences between IAC groups were compared using the $x^{2}$ test. Some patients had multiple nodules, to assess relationships between clinicopathological factors and lung IAC, generalized estimating equation (GEE) was applied for the correlated data using with a logit link function and a binomial distribution. The working correlation structure was selected according to the quasi-likelihood under the independence model criterion minimum principle. In multivariable model 1 , variables with $p<0.1$ in univariable analysis were included. In multivariable model 2 , all independent variables were included to assess the stability of the results. Lastly, a multicollinearity test was performed on all independent variables. All statistical analyses were performed with SPSS Statistics, version 17.0(SPSS Inc., Chicago, IL, USA), and $p<0.05$ was deemed to indicate statistical significance.

\section{Results}

\section{Patient characteristics, radiological features, and} pathological features

In total, 659 pGGNs were detected in 641 patients, of whom 449 were women and 192 were men. The median age was 62.7 years (range 26-78 years). Eight men and 10 women had two pGGNs each. Of the 659 pGGNs $523(79.4 \%)$ were pre-IACs, including 258 (39.2\%) AISs and $265(40.2 \%)$ MIAs. The remaining 136pGGNs (20.6\%) were IACs, including 58 (8.8\%) lepidic predominant, $33(5.0 \%)$ acinar predominant, 43 (6.5\%) papillary predominant, and two $(0.3 \%)$ solid predominant adenocarcinomas. Figure 1 is a flow chart depicting the process ofnodule classification. There were no significant differences in CEA concentration $(p=0.792)$, or the frequencies of pleural retraction $(p=0.857)$, air bronchogram signs $(p=1.000)$, or bubble lucency signs $(p=0.598)$ between the pre-IAC and IAC groups. There were significant differences in the sex distribution $(p=$ $0.025)$, age $(p<0.001)$, CT density $(p<0.001)$, spiculation signs $(p=0.021)$, and mean and maximal diameters (both $p<0.001$ ) between these two groups. The relevant data are summarized in Table 1.

\section{Optimal cutoff values for continuous predictors}

The optimal cutoff values and areas under the curves for age, CEA, maximal and mean diameters, and CTdetermined density for distinguishingbetween pre-IAC and IAC are summarized in Table 2. All data used to derive the sensitivity, specificity, positive predictive, and negative predictive values shown in Table 1 are provided as electronic supplementary material (ESM Table 1). Receiver operating characteristic curve analyses are presented in Fig. 2.

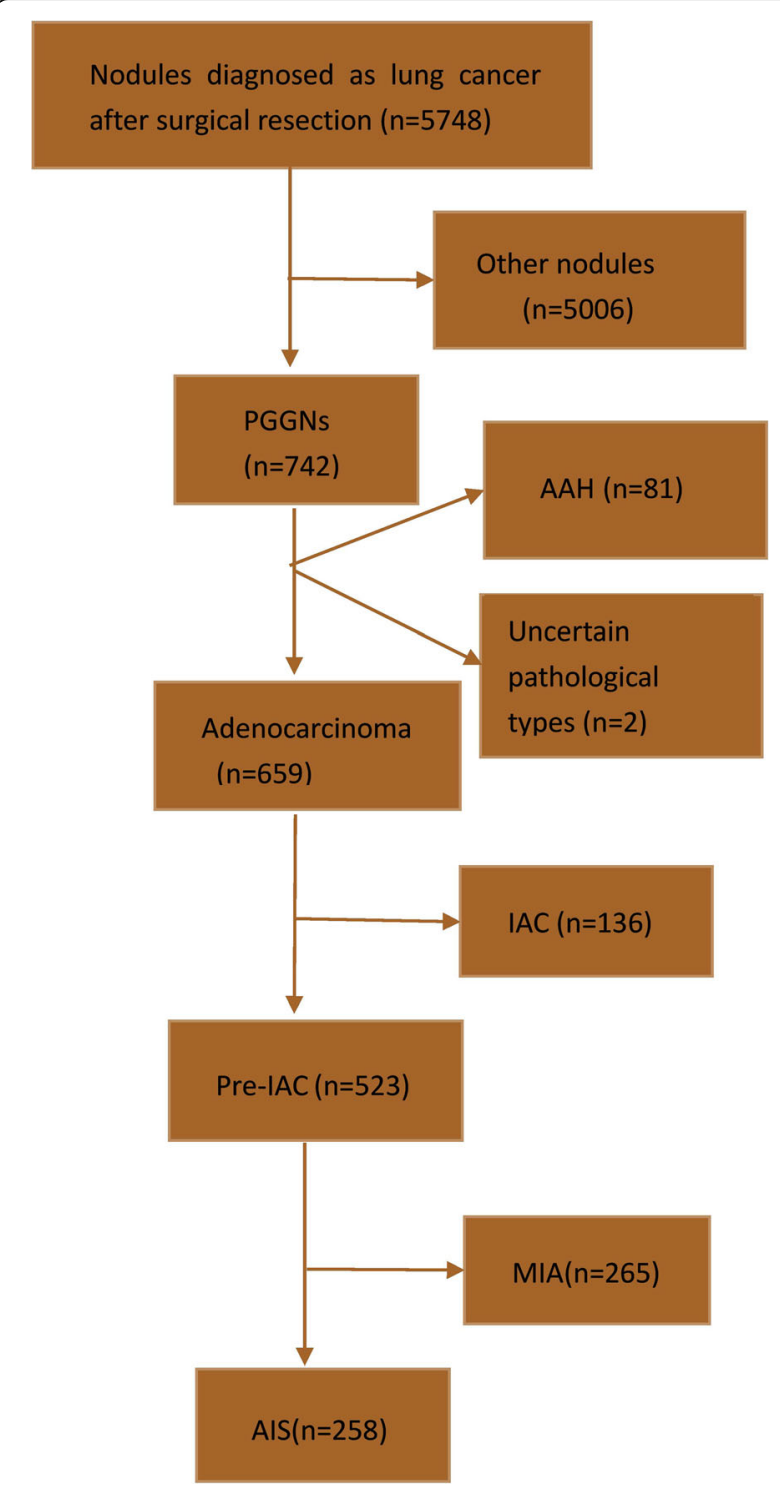

Fig. 1 Flow chart of the classification process

\section{Factors predicting IAC}

The results of the univariable and multivariable analyses are summarized in Table 3. In univariable analysis IAC was significantly associated with age $(p<0.001)$, $\operatorname{sex}(p=$ $0.024)$, mean and maximal diameters (both $p<0.001$ ), CT density $(p<0.001)$, and spiculation signs $(p=0.02)$. Maximal nodule diameters are plotted by pathological type in Fig. 3. Of the 523 pre-IACs, 122 (23.3\%) were $>10 \mathrm{~mm}$ in diameter. Of the 136 IACs, 107 (78.7\%) were $>10 \mathrm{~mm}$ in diameter. With regard to CT density, $226(43.2 \%)$ of the 523 pre-IACs exhibited CT densities > $-600 \mathrm{HU}$, and $89(65.4 \%)$ of the 136 IACs exhibited CT densities > $-600 \mathrm{HU}$. Of the 229 pGGNs that were $>10 \mathrm{~mm}$ in diameter, $122(53.3 \%)$ were pre-IACs (41 AISs and 81 MIAs) and 107 (46.7\%) were 
Table 1 Baseline patient characteristics by diagnostic category

\begin{tabular}{|c|c|c|c|c|c|}
\hline Variable & $\begin{array}{l}\text { Total } \\
(n=659)\end{array}$ & $\begin{array}{l}\text { Pre-IAC } \\
(n=523)\end{array}$ & $\begin{array}{l}\text { IAC } \\
(n=136)\end{array}$ & $x^{2}$ & $p$ \\
\hline Sex & & & & 5.042 & 0.025 \\
\hline Male & $200(30.3 \%)$ & $148(28.3 \%)$ & $52(38.2 \%)$ & & \\
\hline Female & 459 (69.7\%) & 375 (71.7\%) & $84(61.8 \%)$ & & \\
\hline Age, years & & & & 40.540 & $<0.001$ \\
\hline$\leq 55$ & $325(49.3 \%)$ & $291(55.6 \%)$ & $34(25.0 \%)$ & & \\
\hline$>55$ & $334(50.7 \%)$ & $232(44.4 \%)$ & $102(75.0 \%)$ & & \\
\hline Smoking history & & & & 4.985 & 0.723 \\
\hline Never & 350 (53.1\%) & $283(54.1 \%)$ & $67(49.3 \%)$ & & \\
\hline Current or former & 309 (46.9\%) & 240 (45.9\%) & $69(50.7 \%)$ & & \\
\hline Symptoms & & & & 5.742 & 0.831 \\
\hline Absent & $480(72.8 \%)$ & 410 (78.4\%) & $70(51.5 \%)$ & & \\
\hline Present & 179 (27.2\%) & $113(21.6 \%)$ & $66(48.5 \%)$ & & \\
\hline Serum CEA, $\mu \mathrm{g} / \mathrm{L}$ & & & & .069 & 0.792 \\
\hline$<5$ & 652 (99.1\%) & 518 (99.2\%) & $134(98.5 \%)$ & & \\
\hline$\geq 5$ & $6(0.9 \%)$ & $4(0.8 \%)$ & $2(1.5 \%)$ & & \\
\hline Maximal diameter, cm & & & & 145.831 & $<0.001$ \\
\hline$\leq 1$ & $430(65.2 \%)$ & 401 (76.7\%) & $29(21.3 \%)$ & & \\
\hline$>1$ & 229 (34.8\%) & $122(23.3 \%)$ & 107 (78.7\%) & & \\
\hline Mean diameter, $\mathrm{cm}$ & & & & 188.727 & $<0.001$ \\
\hline$\leq 1$ & 475 (72.0\%) & 441 (84.3\%) & $34(25.0 \%)$ & & \\
\hline$>1$ & $184(28.0 \%)$ & $82(15.7 \%)$ & $102(75.0 \%)$ & & \\
\hline $\mathrm{CT}$ density, $\mathrm{HU}$ & & & & 21.374 & $<0.001$ \\
\hline$\leq-600$ & $344(52.2 \%)$ & $297(56.8 \%)$ & 47 (34.6\%) & & \\
\hline$>-600$ & $315(47.8 \%)$ & $226(43.2 \%)$ & $89(65.4 \%)$ & & \\
\hline Pleural retraction & & & & 0.033 & 0.857 \\
\hline Absent & $633(96.1 \%)$ & 502 (96.0\%) & $131(96.3 \%)$ & & \\
\hline Present & $26(3.9 \%)$ & $21(4.0 \%)$ & $5(3.7 \%)$ & & \\
\hline Air bronchogram sign & & & & .000 & 1.00 \\
\hline Absent & 647 (98.2\%) & $513(98.1 \%)$ & $134(98.5 \%)$ & & \\
\hline Present & $12(1.8 \%)$ & $10(1.9 \%)$ & $2(1.5 \%)$ & & \\
\hline Bubble lucency sign & & & & 0.278 & 0.598 \\
\hline Absent & $598(90.7 \%)$ & $473(90.4 \%)$ & 125 (91.9\%) & & \\
\hline Present & $61(9.3 \%)$ & $50(9.6 \%)$ & $11(8.1 \%)$ & & \\
\hline Spiculated sign & & & & 5.329 & 0.021 \\
\hline Absent & $560(85.0 \%)$ & $453(86.6 \%)$ & 107 (78.7\%) & & \\
\hline Present & $99(15.0 \%)$ & $70(13.4 \%)$ & $29(21.3 \%)$ & & \\
\hline
\end{tabular}

CEA carcinoembryonic antigen, CT computed tomography, HU Hounsfield units, IAC invasive adenocarcinoma

IACs (Kruskal-Wallis test $p<0.001$ ). pGGN CT densities are plotted by pathological type in Fig. 4 . Of the 315 nodules with a CT density > $-600 \mathrm{HU}, 226$ (71.7\%) were preIACs (87 AISs and 139 MIAs) and 89 (28.3\%) were IACs (Kruskal-Wallis test $p<0.001$ ).

In multivariable analysis, model 1 which included input variables that had $p<0.1$ in univariable analysis (sex, age, maximal and mean diameters, CT density, and spiculation signs), age $(p<0.001)$, mean diameter $(p=$ $0.002)$, and CT density $(p<0.001)$ were significant independent factors for differentiating pre-IACs and IACs (Table 3). In multivariable analysis, model 2 which included all potential variables and was conducted to assess sensitivity, age $(p<0.001)$, maximal diameter 
Table 2 Optimal cutoff values for continuous predictors

\begin{tabular}{|c|c|c|c|c|c|c|c|}
\hline Indicator & AUC & $p$ & Cut off value ${ }^{\mathbf{a}}$ & Sensitivity & Specificity & PPV & NPV \\
\hline Age (years) & $0.704(0.657,0.751)$ & $<0.001$ & 53 & $52 \%$ & $79 \%$ & $91 \%$ & $30 \%$ \\
\hline Serum CEA ( $\mu \mathrm{g} / \mathrm{L})$ & $0.495(0.440,0.550)$ & 0.862 & 2.19 & $90 \%$ & $15 \%$ & $80 \%$ & $28 \%$ \\
\hline Max diameter (mm) & $0.845(0.805,0.885)$ & $<0.001$ & 10.78 & $81 \%$ & $79 \%$ & $94 \%$ & $52 \%$ \\
\hline $\mathrm{CT}$ value $(\mathrm{HU})$ & $0.664(0.613,0.715)$ & $<0.001$ & -582.28 & $66 \%$ & $60 \%$ & $87 \%$ & $32 \%$ \\
\hline Mean diameter (mm) & $0.852(0.813,0.89)$ & $<0.001$ & 10.09 & $86 \%$ & $75 \%$ & $93 \%$ & $58 \%$ \\
\hline
\end{tabular}

${ }^{a}$ Cutoff values were determined via the maximal Youden index (sensitivity + specificity -1).AUC area under the receiver operating characteristic curve, CEA carcinoembryonic antigen, $C T$ computed tomography, HU Hounsfield units, Max maximal, NPV negative predictive value, PPV positive predictive value

$(p=0.032)$, mean diameter $(p=0.002)$, and CT density $(p<0.001)$ were significant independent factors for distinguishing between pre-IACs and IACs (Table 3). A multicollinearity test indicated no multicollinearity problems in the multivariable models (Table 4).

All univariable and multivariable analysis results are presented as a forest plot in Fig. 5. Age, maximal diameter, mean diameter, and CT density were all independent factors that distinguished IAC from pre-IAC.

\section{Recurrence of pGGNs}

After a median follow-up interval of 41 months (range 7-52 months) after resection, no pGGN IACs or pre-
IACs had recurred. After resection, chest $\mathrm{CT}$ scans were periodically conducted and no new nodules were detected.

\section{Discussion}

Several studies have analyzed relationships between CT features of pGGNs and pathological type [15-18]. However, to the best of our knowledge, no published study has identified predictors of lung IACs in pGGNs by analyzing their clinical and imaging characteristics. The present study investigated 659 adenocarcinomas manifesting as pGGNs on CT; thus, making the study was

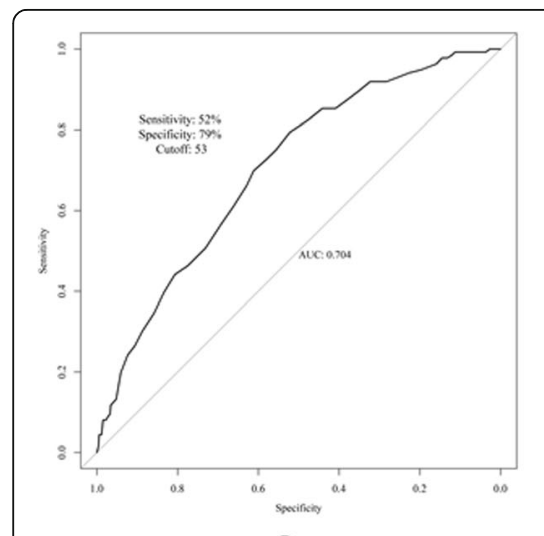

a
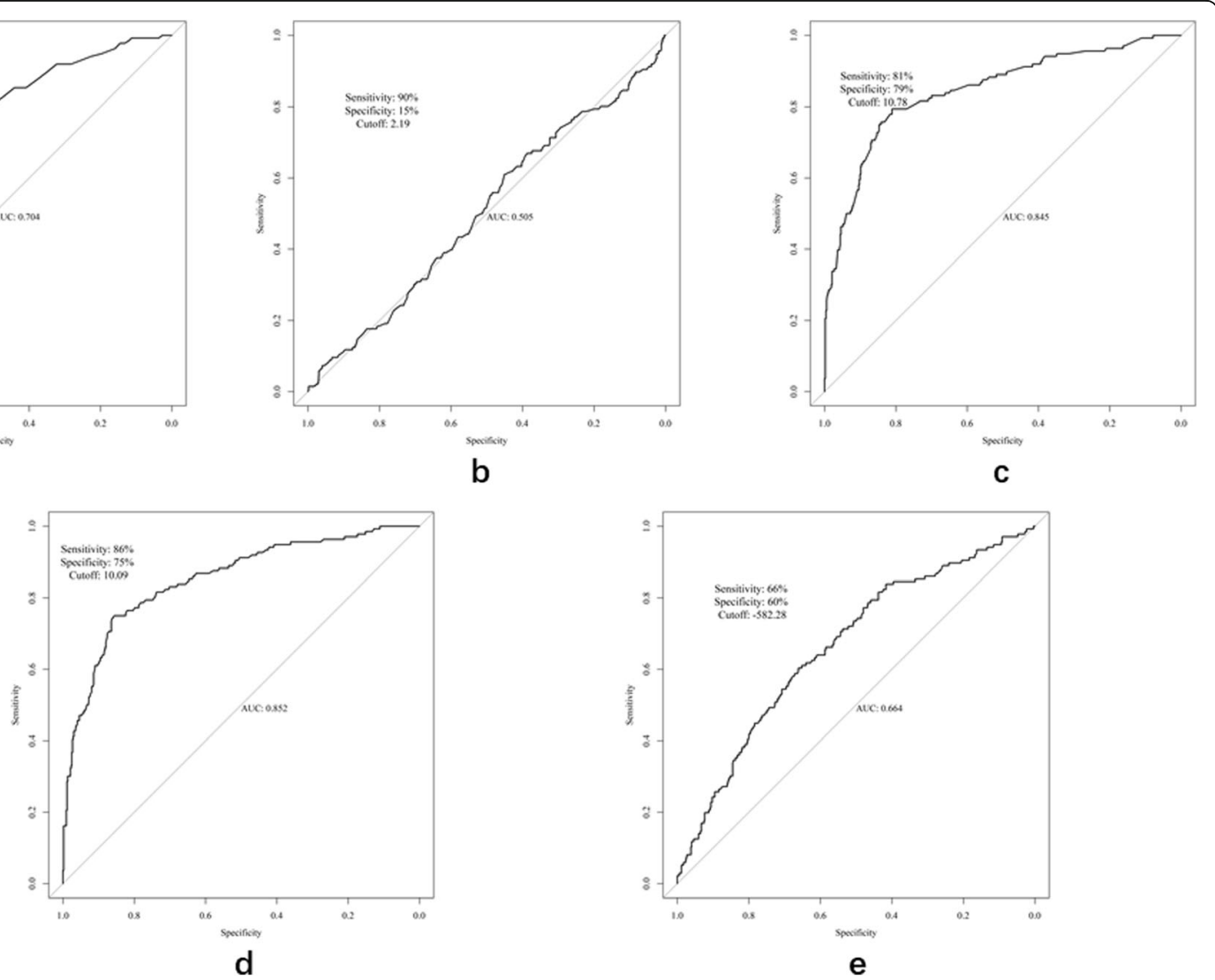

Fig. 2 Receiver operating characteristic curves for age (a), serum carcinoembryonic antigen concentration (b), maximal diameter (c), mean diameter (d), and computed tomography-determined density (e). AUC, area under the receiver operating characteristic curve 
Table 3 Univariable and multivariable analysis of associations between invasive adenocarcinoma and potential predictors thereof. Working correlation structure in the generalized estimation model was determined via quasi-likelihood under the independence model criterion, and unstructured working correlation structure was selected. Multivariable model 1 only included variables whose $p$ values were $<0.1$ in the univariable model. Multivariable model 2 included all potential variables, and was performed as a sensitivity analysis

\begin{tabular}{|c|c|c|c|c|c|c|c|}
\hline \multirow{2}{*}{ Characteristics } & & \multicolumn{2}{|l|}{ Univariable model } & \multicolumn{2}{|l|}{ Multivariable Model 1} & \multicolumn{2}{|l|}{ Multivariable Model 2} \\
\hline & & $\overline{\mathrm{OR}}(95 \% \mathrm{Cl})$ & $p$ & $\overline{\mathrm{OR}}(95 \% \mathrm{Cl})$ & $p$ & $\overline{\mathrm{OR}(95 \% \mathrm{Cl})}$ & $p$ \\
\hline \multirow[t]{2}{*}{ Sex } & Female & $0.634(0.427,0.942)$ & 0.024 & $0.784(0.468,1.313)$ & 0.356 & $0.708(0.415,1.208)$ & 0.205 \\
\hline & Male & Reference & & Reference & & Reference & \\
\hline \multirow[t]{2}{*}{ Age, years } & $>55$ & $3.764(2.464,5.748)$ & $<0.001$ & $2.240(1.370,3.665)$ & 0.001 & $2.732(1.605,4.651)$ & $<0.001$ \\
\hline & $\leq 55$ & Reference & & Reference & & Reference & \\
\hline \multirow[t]{2}{*}{ Smoking history } & Current or former & $0.801(0.590,1.284)$ & 0.722 & - & - & $0.721(0.401,1.023)$ & 0.942 \\
\hline & Never & Reference & & Reference & & Reference & \\
\hline \multirow[t]{2}{*}{ Symptoms } & Present & $1.080(0.780,2.196)$ & 0.830 & - & - & $0.988(0.697,2.010)$ & 0.984 \\
\hline & Absent & Reference & & Reference & & Reference & \\
\hline \multirow[t]{2}{*}{ Serum CEA, $\mu \mathrm{g} / \mathrm{L}$} & $>1$ & $1.932(0.350,10.661)$ & 0.45 & - & - & $0.967(0.235,3.978)$ & 0.963 \\
\hline & $\leq 1$ & Reference & & Reference & & Reference & \\
\hline \multirow[t]{2}{*}{ Maximal diameter, $\mathrm{cm}$} & $>1$ & $13.058(8.192,20.814)$ & $<0.001$ & $2.690(0.994,7.276)$ & 0.051 & $2.992(1.098,8.153)$ & 0.032 \\
\hline & $\leq 1$ & Reference & & Reference & & Reference & \\
\hline \multirow[t]{2}{*}{ Mean diameter, $\mathrm{cm}$} & $>1$ & $16.190(10.285,25.486)$ & $<0.001$ & $6.207(2.352,16.383)$ & $<0.001$ & $4.707(1.761,12.583)$ & 0.002 \\
\hline & $\leq 1$ & Reference & & Reference & & Reference & \\
\hline \multirow[t]{2}{*}{$\mathrm{CT}$ density, $\mathrm{HU}$} & $>-600$ & $2.612(1.756,3.885)$ & $<0.001$ & $3.542(2.155,5.822)$ & $<0.001$ & $3.886(2.307,6.548)$ & $<0.001$ \\
\hline & $\leq-600$ & Reference & & Reference & & Reference & \\
\hline \multirow[t]{2}{*}{ Pleural retraction } & Present & $0.991(0.329,2.986)$ & 0.988 & - & - & $2.042(0.444,9.390)$ & 0.359 \\
\hline & Absent & Reference & & Reference & & Reference & \\
\hline \multirow[t]{2}{*}{ Air bronchogram sign } & Present & $0.765(0.166,3.535)$ & 0.732 & - & - & $0.193(0.042,0.882)$ & 0.034 \\
\hline & Absent & Reference & & Reference & & Reference & \\
\hline \multirow[t]{2}{*}{ Bubble lucency sign } & Present & $0.824(0.415,1.639)$ & 0.582 & - & - & $0.583(0.249,1.365)$ & 0.214 \\
\hline & Absent & Reference & & Reference & & Reference & \\
\hline \multirow[t]{2}{*}{ Spiculated sign } & Present & $1.761(1.093,2.838)$ & 0.02 & $1.445(0.781,2.671)$ & 0.241 & $1.758(0.880,3.511)$ & 0.11 \\
\hline & Absent & Reference & & Reference & & Reference & \\
\hline
\end{tabular}

CEA carcinoembryonic antigen, $C I$ confidence interval, $C T$ computed tomography, HU Hounsfield units, IAC invasive adenocarcinoma, OR odds ratio (estimated from generalized estimation model)

larger than most recent studies regarding pathological classification of pGGNs [11, 15, 17, 19, 20].

Kitami et al. [15] reported cutoffs of $-600 \mathrm{HU}$ for CT density and $10 \mathrm{~mm}$ for maximum diameter for distinguishing between IAC and non-IAC pGGNs of the lung. The corresponding cutoffs identified in the current study are similar to those values, and other previously reported values [11]. Because integers are more convenient in clinical practice, we used published cutoff values in our univariable and multivariable analyses (age $>55$ years, maximal diameter $>1 \mathrm{~cm}, \mathrm{CT}$ density $>-600 \mathrm{HU}$ ). In the present study, model 2 suggested that maximal and mean diameters could reliably and independently distinguish IACs in patients with pGGNs with diameters of $<3 \mathrm{~cm}$; this finding was consistent with reported literature $[11,15]$. In the current study, a tumor diameter of $>10 \mathrm{~mm}$ was a significant independent predictor of IAC; however, that result is not readily apparent from the scatter plot shown in Fig. 3. The best predictor of IAC may be a diameter of $>20 \mathrm{~mm}$, because only one of the pre-IACs shown in Fig. 3 had a diameter $>20 \mathrm{~mm}$. Notably, that threshold yields a high positive predictive value; however, the corresponding negative predictive value would be abysmal. This would result in a high rate of missed diagnoses in clinical practice. Importantly, the observation in the present study that a tumor diameter of $>10 \mathrm{~mm}$ was an independent predictor of IAC is consistent with results reported in similar previous studies [17, 18, 21-23]. As depicted in the scatter plot shown in Fig. 3, a more aggressive histological subtype tended to be associated with larger pGGNdiameter. Thus, tumor size is the most important 


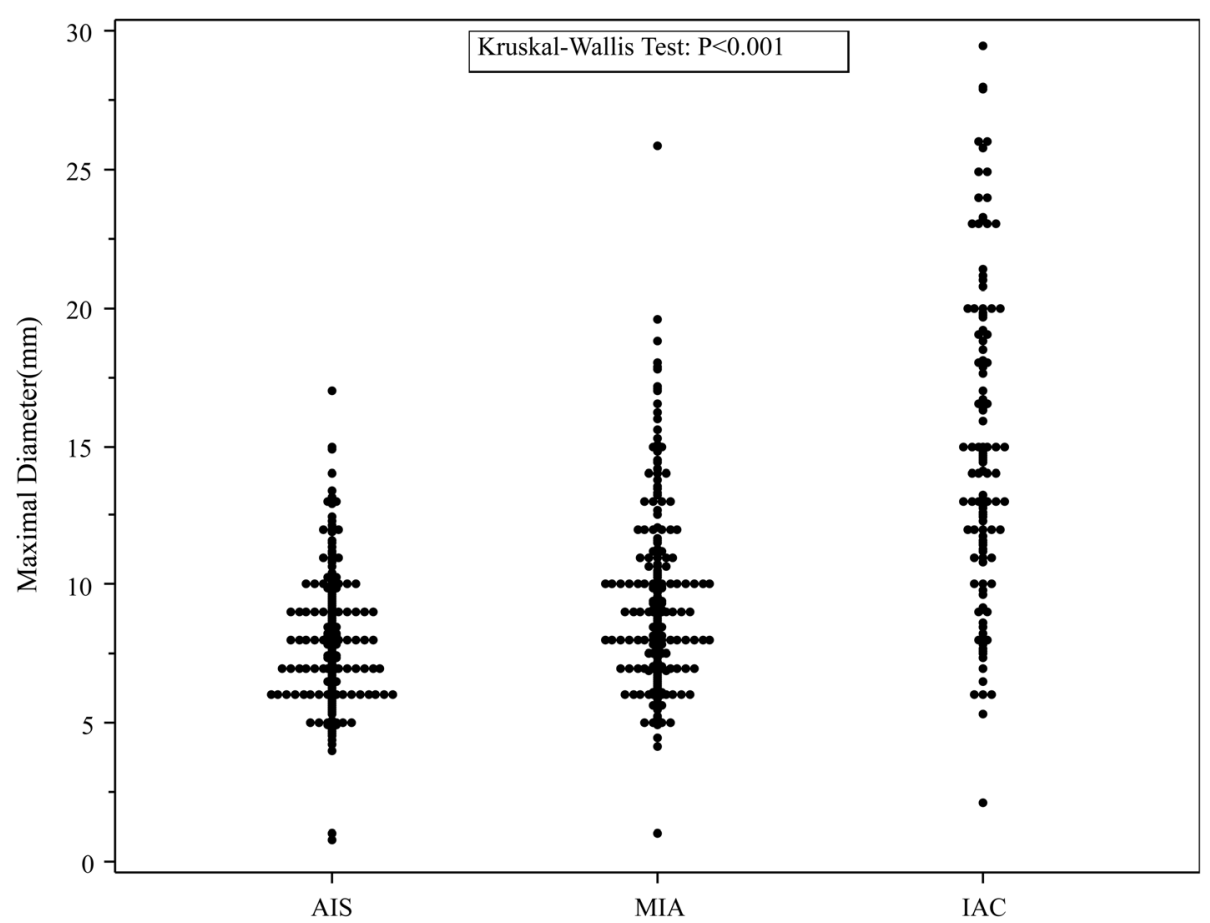

Fig. 3 Scatter plot of pure ground-glass nodule maximal diameters plotted by pathological type (AIS, adenocarcinoma in situ; IAC, invasive adenocarcinoma; MIA, minimally invasive adenocarcinoma)

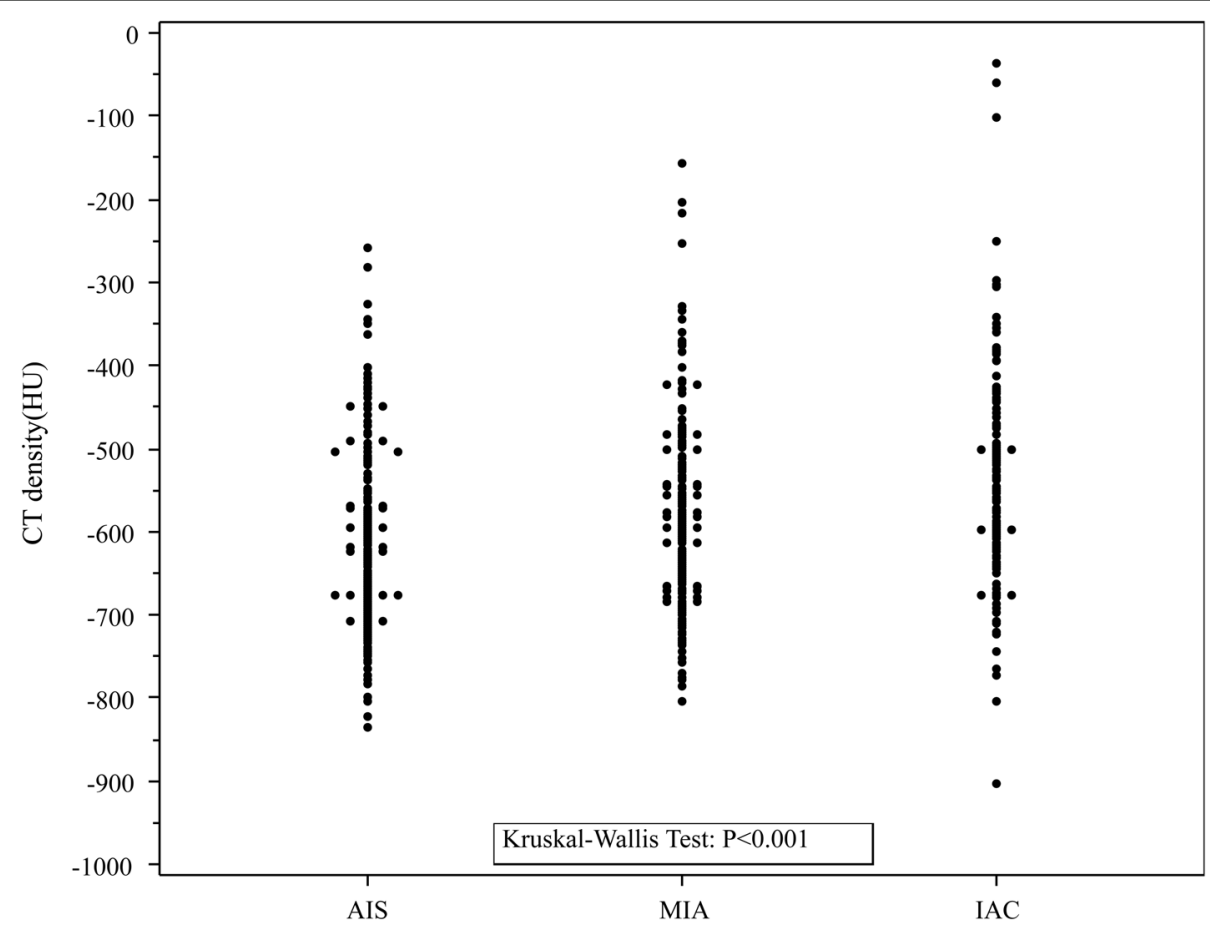

Fig. 4 Scatter plot of computed tomography-determined density of pure ground-glass nodules plotted by pathological type (AIS, adenocarcinoma in situ; CT, computed tomography; HU, Hounsfield units; IAC, invasive adenocarcinoma; MIA, minimally invasive adenocarcinoma) 
Table 4 Multicollinearity test from multivariable models 1 and 2

\begin{tabular}{llll}
\hline Index & Criterion & Multivariable Model 1 & Multivariable Model 2 \\
\hline Conditional index & $<10$ & 6.52 & 6.48 \\
Minimal tolerance & $>0.1$ & 0.26 & 0.27 \\
Maximal variance inflation factor & $<10$ & 3.83 & 3.69 \\
\hline
\end{tabular}

variable on which to base decisions pertaining to the management of pGGNs [12, 24, 25].

Although nodules $>10 \mathrm{~mm}$ in diameter are more likely to be IACs, smaller nodules may also be IACs. In the present study 29 nodules with diameters of $<10 \mathrm{~mm}$ were found to be IACs. Persistent nodules larger than 5 $\mathrm{mm}$ should be followed for at least 4 years. And growth of more than $2 \mathrm{~mm}$ in maximal diameter is considered significant [26]. It is appropriate to monitor nodules with diameters $<10 \mathrm{~mm}$ independently. With regard to these smaller nodules, further research is needed to establish a basis for clinical planning.

Although CT-determined density of pGGNs of $>-600$ HU was a significant predictor of IAC in the present study, the scatter plot shown in Fig. 4 does not clearly depict this result. We presume that the use ofa single factor to predict IACs is not good clinical practice; simultaneous assessment of multiple factors would be more accurate. Notably, Kitami et al. [15] reported that the CT-determined density of pGGNs can distinguish IACs, and Lim et al. [11] reported that pGGN density was a significant predictor of tumor invasiveness. In contrast, Heidinger et al. [17] reported that pGGN density was not significantly associated with pathological diagnosis, and several other groups have also reported no significant differences in nodule density between AISs, MIAs, and IACs manifesting as pGGNs on CT $[20,21]$. All of these studies were carried out with similar sampling methods. Thus, whether CT density is a valid parameter for distinguishing IACs remains controversial. CT density should be combined with other indicators such as size, patient age, and certain CT signs when predicting the nature of a lesion preoperatively.

\begin{tabular}{|c|c|c|c|c|c|c|c|}
\hline Characteristic & Total & Pre-IAC & IAC & & Method & OR(95\% CI) & $\mathbf{P}$ \\
\hline Overall & $659(100 \%)$ & $523(79.36 \%)$ & $136(20.64 \%)$ & & & & \\
\hline \multicolumn{8}{|l|}{ Sex } \\
\hline Male & $200(30.35 \%)$ & $148(28.30 \%)$ & $52(38.24 \%)$ & a & Univariable & $0.634(0.427,0.942)$ & 0.024 \\
\hline Female & $459(69.65 \%)$ & $375(71.70 \%)$ & $84(61.76 \%)$ & $=$ & Multivariable & $0.708(0.415,1.208)$ & 0.205 \\
\hline \multicolumn{8}{|l|}{ Age } \\
\hline$<=55$ & $325(49.32 \%)$ & $291(55.64 \%)$ & $34(25.00 \%)$ & $\longrightarrow$ & Univariable & $3.764(2.464,5.748)$ & $<0.001$ \\
\hline$>55$ & $334(50.68 \%)$ & $232(44.36 \%)$ & $102(75.00 \%)$ & $\longrightarrow$ & Multivariable & $2.732(1.605,4.651)$ & $<0.001$ \\
\hline \multicolumn{8}{|l|}{ CEA } \\
\hline$<=5$ & $652(99.09 \%)$ & $518(99.23 \%)$ & $134(98.53 \%)$ & $\longrightarrow$ & Univariable & $1.932(0.350,10.661)$ & 0.450 \\
\hline$>5$ & $6(0.91 \%)$ & $4(0.77 \%)$ & $2(1.47 \%)$ & $\rightarrow$ & Multivariable & $0.967(0.235,3.978)$ & 0.963 \\
\hline \multicolumn{8}{|l|}{ Maximal diameter } \\
\hline$<=1$ & $430(65.25 \%)$ & $401(76.67 \%)$ & $29(21.32 \%)$ & $\longrightarrow$ & Univariable & $13.058(8.192,20.814)$ & $<0.001$ \\
\hline$>1$ & $229(34.75 \%)$ & $122(23.33 \%)$ & $107(78.68 \%)$ & - & Multivariable & $2.992(1.098,8.153)$ & 0.032 \\
\hline \multicolumn{8}{|l|}{ Mean diameter } \\
\hline$<=1$ & $475(72.08 \%)$ & $441(84.32 \%)$ & $34(25.00 \%)$ & $\Rightarrow$ & Univariable & $16.190(10.285,25.486)$ & $<0.001$ \\
\hline$>1$ & $184(27.92 \%)$ & $82(15.68 \%)$ & $102(75.00 \%)$ & $\longrightarrow$ & Multivariable & $4.707(1.761,12.583)$ & 0.002 \\
\hline \multicolumn{8}{|l|}{ CT density } \\
\hline$<=-600$ & $344(52.20 \%)$ & $297(56.79 \%)$ & $47(34.56 \%)$ & $\rightarrow$ & Univariable & $2.612(1.756,3.885)$ & $<0.001$ \\
\hline$>-600$ & $315(47.80 \%)$ & $226(43.21 \%)$ & $89(65.44 \%)$ & $\longrightarrow$ & Multivariable & $3.886(2.307,6.548)$ & $<0.001$ \\
\hline \multicolumn{8}{|l|}{ Pleural retraction } \\
\hline No & $633(96.05 \%)$ & $502(95.98 \%)$ & $131(96.32 \%)$ & $\rightarrow$ & Univariable & $0.991(0.329,2.986)$ & 0.988 \\
\hline Yes & $26(3.95 \%)$ & $21(4.02 \%)$ & $5(3.68 \%)$ & - & Multivariable & $2.042(0.444,9.390)$ & 0.359 \\
\hline \multicolumn{8}{|c|}{ Air bronchogram sign } \\
\hline No & $647(98.18 \%)$ & $513(98.09 \%)$ & $134(98.53 \%)$ & $\rightarrow$ & Univariable & $0.765(0.166,3.535)$ & 0.732 \\
\hline Yes & $12(1.82 \%)$ & $10(1.91 \%)$ & $2(1.47 \%)$ & - & Multivariable & $0.193(0.042,0.882)$ & 0.034 \\
\hline \multicolumn{8}{|c|}{ Bubble lucency sign } \\
\hline No & $598(90.74 \%)$ & $473(90.44 \%)$ & $125(91.91 \%)$ & $=$ & Univariable & $0.824(0.415,1.639)$ & 0.582 \\
\hline Yes & $61(9.26 \%)$ & $50(9.56 \%)$ & $11(8.09 \%)$ & $=$ & Multivariable & $0.583(0.249,1.365)$ & 0.214 \\
\hline \multicolumn{8}{|l|}{ Spiculated sign } \\
\hline No & $560(84.98 \%)$ & $453(86.62 \%)$ & $107(78.68 \%)$ & -- & Univariable & $1.761(1.093,2.838)$ & 0.020 \\
\hline Yes & $99(15.02 \%)$ & $70(13.38 \%)$ & $29(21.32 \%)$ & 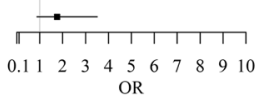 & Multivariable & $1.758(0.880,3.511)$ & 0.110 \\
\hline
\end{tabular}


Liu et al. [22] reported that the presence of signs of spiculation is suggestive of a diagnosis of IAC. In univariable analysis in the present study spiculation was a significant predictor of IAC; however, this was not confirmed in multivariable analysis. Spiculation is considered to be evidence of malignancy and to represent invasiveness. In one study spiculation was the strongest predictor of invasion [27].

In the present study age was a significant predictor of IAC. To the best of our knowledge no previous studies have identified this correlation. This hitherto unreported result of the current study warrants further investigation in prospective studies. In clinical practice, the recommendation of surgical resection in patients with pGGN who are aged $>55$ years is determined with respect to a combination of other additional factors such as nodule diameter and nodule density. IACs presenting as pGGNs have a good prognosis. In the present study, no pGGN IACs recurred after surgical resection during a median follow-up interval of 41 months (range, 7-52 months); this finding is consistent with the results of previous investigations $[28-30]$.

IACs and pre-IACs are known to require treatment involving different surgical procedures. The risk of lung cancer is high if the nonsolid nodule size is greater than $8 \mathrm{~mm}$ [31]. At our hospital surgery is performed if the diameter of the pGGN is $>8 \mathrm{~mm}$. Most patients are treated via limited resection and frozen section, and lobectomy is performed if an invasive component is detected via frozen section. Unfortunately, the accuracy of frozen section is not satisfactory [32]. Moreover, distinction between MIA and IAC remains difficult, regardless of the presence of invasive components. Because the accuracy of intraoperative frozen sections is not entirelyclear, it is easier to determine the appropriate treatment if IAC can be predicted accurately. Although limited resection is the preferred treatment for pGGN, it may not be possible to remove all lesions. In the present study $20.6 \%$ of pGGNs were eventually diagnosed as IACs, therefore the presence of a pGGN should not be used as an indication for limited resection.

The current study had some limitations. First, it was retrospective, and all the data were derived from a single institution. However, all data in the study were collected during 2016 and all patients were managed in accordance with the same protocol; thus, there was conceivably relatively minimal bias. Second, this study only included patients in whom a diagnosis had been established via resection, whereas some unresected pGGNs may also have been adenocarcinomas. These factors may have contributed to a selection bias in the present study. A prospective study would be required to minimize these potential sources of bias. Third, distinguishing between pGGNs and other nodules is subjective in this study.
Two reviewers (a radiologist and a surgeon) evaluated all CT scans independently to minimize this source of bias. Finally, blood vessels and bronchioles could not always be fully excluded when the boundaries of lesions were delineated, this may have contributed to variations between observers in nodule measurements and lesion characterization.

\section{Conclusions}

Patients with pGGNs $<3 \mathrm{~cm}$ in diameter on CT are more likely to have IACs if they are aged $>55$ years, exhibit a nodule diameter $>1 \mathrm{~cm}$, and have a CTdetermined nodule density $>-600 \mathrm{HU}$. Postoperatively, IACs initially identified as pGGNs have a good prognosis, and there were no recurrences during a median follow-up interval of 41 months in the current study. The results of this study may assist decisions pertaining to the selection of surgical procedures in patients with pGGNs identified as being at high risk of malignant disease.

\section{Supplementary information}

Supplementary information accompanies this paper at https://doi.org/10. 1186/s13014-020-01628-x

\section{Additional file 1.}

\section{Abbreviations}

pGGNs: Pure ground-glass nodules; CT: Computed tomography;

IACs: Invasive adenocarcinoma; HU: Hounsfield units; AIS: Adenocarcinoma in situ; MIA: Minimally invasive adenocarcinoma; CEA: Serum carcinoembryonic antigen

\section{Acknowledgments}

We thank Dr. Owen Proudfoot and Dr. Ryan Chastain-Gross from Liwen Bianji, Edanz Editing China (www.liwenbianji.cn/ac) for editing the English text of a draft of this manuscript.

\section{Authors' contributions}

(I) Conception and design: Huan-Huan Yang, Yi-Lv LV, Bo Ye, Ding-Zhong Hu (II) Administrative support: Ding-Zhong Hu, Bo Ye. (III) Provision of study materials or patients: Huan-Huan Yang, Yi-Lv Lv, Xing-Hai Fan, Zhi-Yong Ai, XiuChun Xu. (IV) Collection and assembly of data: Huan-Huan Yang, Yi-Lv LV, Xing-Hai Fan. (V) Data analysis and interpretation: Huan-Huan Yang, Yi-Lv LV, Xing-Hai Fan, Zhi-Yong Ai, Xiu-Chun Xu. (VI) Manuscript writing: All authors. (VII) Final approval of manuscript: All authors.

\section{Funding}

This work was supported by the Program in Western Medicine approved by Shanghai Science and Technology Commission (16411966000), the Interdisciplinary Program of Shanghai Jiaotong University (YG2014QN22), Science and Technology Innovation Sepcial Funding project of Shanghai Baoshan District (19-E-44) and Natural Science Foundation of China (81402571).

Availability of data and materials

The datasets used and/or analysed during the current study are available from the corresponding author on reasonable request.

Ethics approval and consent to participate

The study was conducted in accordance with the Declaration of Helsinki. The Institutional Review Board and the Ethics Committee of Shanghai Chest 
Hospital approved the study, and waived the requirement for patient consent due to its retrospective design.

\section{Consent for publication}

Not applicable.

\section{Competing interests}

The authors declare that they have no competing interests.

\section{Author details}

'Department of ThoracicSurgery, Shanghai Chest Hospital, Shanghai Jiaotong University, Shanghai 200030, China. ${ }^{2}$ Department of Respiratory Medicine, Shanghai Zhongye Hospital, Shanghai 200941, China.

Received: 9 May 2020 Accepted: 21 July 2020

Published online: 31 July 2020

\section{References}

1. Matsuguma H, Mori K, Nakahara R, et al. Characteristics of subsolid pulmonary nodules showing growth during follow-up with CT scanning. Chest. 2012;143(2):436-43.

2. Naidich DP, et al. Recommendations for the management of subsolid pulmonary nodules detected at CT: a statement from the Fleischner society. Radiology. 2013;266(1):304-17.

3. Park CM, et al. CT findings of atypical adenomatous hyperplasia in the lung. Korean J Radiol. 2006;7(2):80-6.

4. Kim HY, et al. Persistent pulmonary nodular ground-glass opacity at thinsection CT: histopathologic comparisons. Radiology. 2007;245(1):267-75.

5. Noguchi $M$, et al. Small adenocarcinoma of the lung. Histologic characteristics and prognosis. Cancer. 1995;75(12):2844-52.

6. Yim J, Zhu LC, Chiriboga L, Watson HN, Goldberg JD, Moreira AL. Histologic features are important prognostic indicators in early stages lung adenocarcinomas. Mod Pathol. 2007;20:233-41.

7. Borczuk $A C$, et al. Invasive size is an independent predictor of survival in pulmonary adenocarcinoma. Am J Surg Pathol. 2009;33(3):462-9.

8. Maeshima AM, et al. Histological scoring for small lung adenocarcinomas 2 cm or less in diameter: a reliable prognostic indicator. J Thorac Oncol. 2010; 5(3):333-9.

9. Zhang J, et al. Why do pathological stage IA lung adenocarcinomas vary from prognosis?: a clinicopathologic study of 176 patients with pathological stage IA lung adenocarcinoma based on the IASLC/ATS/ERS classification. J Thorac Oncol. 2013;8(9):1196-202.

10. Nakata M, et al. Focal ground-glass opacity detected by low-dose helical CT. Chest. 2002;121(5):1464-7.

11. Lim HJ, et al. Persistent pure ground-glass opacity lung nodules $>/=10 \mathrm{~mm}$ in diameter at CT scan: histopathologic comparisons and prognostic implications. Chest. 2013:144(4):1291-9.

12. MacMahon $\mathrm{H}$, et al. Guidelines for Management of Incidental Pulmonary Nodules Detected on CT images: from the Fleischner society 2017. Radiology. 2017;284(1):228-43.

13. Travis WD, et al. Introduction to the 2015 World Health Organization classification of tumors of the lung, pleura, Thymus, and heart. J Thorac Oncol. 2015;10(9):1240-2.

14. Travis WD, et al. International Association for the Study of Lung Cancer/ American Thoracic Society/European Respiratory Society: international multidisciplinary classification of lung adenocarcinoma: executive summary. Proc Am Thorac Soc. 2011;8(5):381-5.

15. Kitami A, et al. Correlation between histological invasiveness and the computed tomography value in pure ground-glass nodules. Surg Today. 2016:46(5):593-8.

16. Hwang IP, et al. Persistent pure ground-glass nodules larger than $5 \mathrm{~mm}$ : differentiation of invasive pulmonary adenocarcinomas from Preinvasive lesions or minimally invasive adenocarcinomas using texture analysis. Investig Radiol. 2015;50(11):798-804

17. Heidinger $\mathrm{BH}$, et al. Lung adenocarcinoma manifesting as pure groundglass nodules: correlating CT size, volume, density, and roundness with Histopathologic invasion and size. J Thorac Oncol. 2017;12(8):1288-98.

18. Dai $C$, et al. Clinical and radiological features of synchronous pure groundglass nodules observed along with operable non-small cell lung cancer. J Surg Oncol. 2016;113(7):738-44.
19. Jin X, et al. CT characteristics and pathological implications of early stage (T1NOM0) lung adenocarcinoma with pure ground-glass opacity. Eur Radiol. 2015;25(9):2532-40.

20. Xiang $W$, et al. Morphological factors differentiating between early lung adenocarcinomas appearing as pure ground-glass nodules measuring $</=$ $10 \mathrm{~mm}$ on thin-section computed tomography. Cancer Imaging. 2014;14:33.

21. Lee SM, Park CM, Goo JM, Lee HJ, Wi JY, Kang CH. Invasive pulmonary adenocarcinomas versus preinvasive lesions appearing as ground-glass nodules: differentiation by using CT features. Radiology. 2013;268:265-73.

22. Liu LH, et al. CT findings of persistent pure ground glass opacity: can we predict the invasiveness? Asian Pac J Cancer Prev. 2015;16(5):1925-8.

23. Ding $\mathrm{H}$, et al. Value of $\mathrm{CT}$ characteristics in predicting invasiveness of adenocarcinoma presented as pulmonary ground-glass nodules. Thorac Cardiovasc Surg. 2017;65(2):136-41.

24. Callister ME, et al. British Thoracic Society guidelines for the investigation and management of pulmonary nodules. Thorax. 2015;70(Suppl 2):ii1-ii54.

25. Gould MK, et al. Evaluation of individuals with pulmonary nodules: when is it lung cancer? Diagnosis and management of lung cancer, 3rd ed: American College of Chest Physicians evidence-based clinical practice guidelines. Chest. 2013;143(5 Suppl):e93S-e120S.

26. Pedersen JH, Saghir Z, Wille MMW, et al. Ground-glass opacity lung nodules in the Era of lung cancer $\mathrm{CT}$ screening: radiology, pathology, and clinical management. Oncology. 2016;30(3):266

27. Lee HY, Lee KS. Ground-glass opacity nodules: histopathology, imaging evaluation, and clinical implications. J Thorac Imaging. 2011;26:106-18.

28. Cho S, et al. Pathology and prognosis of persistent stable pure ground-glass opacity nodules after surgical resection. Ann Thorac Surg. 2013;96(4):1190-5.

29. Lee HY, et al. Pure ground-glass opacity neoplastic lung nodules: histopathology, imaging, and management. AJR Am J Roentgenol. 2014; 202(3):W224-33

30. Ohtsuka T, et al. A clinicopathological study of resected pulmonary nodules with focal pure ground-glass opacity. Eur J Cardiothorac Surg 2006:30(1):160-3.

31. van Klaveren RJ, Oudkerk M, Prokop M, et al. Management of lung nodules detected by volume CT scanning. N Engl J Med. 2009;361:2221-9.

32. Yeh YC, Nitadori J, Kadota K, et al. Using frozen section to identify histological patterns in stage I lung adenocarcinoma of $</=3 \mathrm{~cm}$ : accuracy and interobserver agreement [J]. Histopathology. 2015;66(7):922-38. https:// doi.org/10.1111/his.12468

\section{Publisher's Note}

Springer Nature remains neutral with regard to jurisdictional claims in published maps and institutional affiliations.

Ready to submit your research? Choose BMC and benefit from:

- fast, convenient online submission

- thorough peer review by experienced researchers in your field

- rapid publication on acceptance

- support for research data, including large and complex data types

- gold Open Access which fosters wider collaboration and increased citations

- maximum visibility for your research: over $100 \mathrm{M}$ website views per year

At BMC, research is always in progress.

Learn more biomedcentral.com/submission 